This document is published in:

Pediatric Dermatology, Vol. 30, no 6 (2013), pp. 125-131

DOI: http://dx.doi.org/10.1111/j.1525-1470.2012.01748.x

(C) 2012 Wiley Periodicals Inc. 


\title{
Epidermolysis Bullosa Simplex with Mottled Pigmentation: A Family Report and Review
}

\author{
Begoña Echeverría-García, M.D.,* Asunción Vicente, M.D.,† Ángela Hernández, M.D.,* \\ Jose M. Mascaró, M.D., Isabel Colmenero, M.D.,* Ana Terrón, M.D.,§ \\ María J. Escámez, M.D., Marcela del Río, M.D.,** Maria A. González-Enseñat, M.D., † and \\ Antonio Torrelo, M.D.* \\ *Department of Dermatology, Hospital Infantil Universitario Niño Jesus, Madrid, Spain, †Dermatology Service, \\ Hospital San Juan de Dios, Barcelona, \$Department of Dermatology, Hospital Clínico, Barcelona, Spain, §East of \\ Scotland Regional Genetics Service, Ninewells Hospital, Dundee, UK, $\uparrow$ Regenerative Medicine Unit, Epithelial \\ Biomedicine Division, Centro de Investigaciones Energéticas, Medioambientales y Tecnológicas (CIEMAT) \\ Madrid, **Department of Bioengineering, Universidad Carlos III, Madrid, Spain
}

\begin{abstract}
Epidermolysis bullosa simplex with mottled hyperpigmentation (EBS-MP) is an uncommon subtype of EBS. Its clinical features depend on the age of diagnosis, and clinical variations have been described even within family members. We present six cases from two unrelated Spanish families each with several affected members with EBS-MP and review the clinical and genetic findings in all reported patients. We highlight the changing clinical features of the disease throughout life.
\end{abstract}

Epidermolysis bullosa simplex with mottled hyperpigmentation (EBS-MP) is a rare subtype of EBS (Online Mendelian Inheritance in Man [OMIM] no. 131960). Since its first description in 1979 (1), multiple families and some sporadic cases have been reported from all over the world. Clinical heterogeneity regarding skin features and age of presentation is recognized, as well as additional manifestations such as photosensitivity (2) and dental disorders $(1,3)$. Classic clinical features in most patients include serous, nonscarring blisters on the distal part of the extremities and slowly progressing reticular hyperpigmentation. Blisters usually appear at birth or in early infancy, tend to decrease with age, and only occasionally appear in adults, after minor trauma or friction. The hyperpigmented lesions appear later in infancy or

Address correspondence to Begoña Echeverría García, M.D., Department of Dermatology, Hospital Infantil Universitario Niño Jesus, Avenida Menéndez Pelayo, 6528009 Madrid, Spain, or e mail: begoecheverria2@gmail.com. childhood, spread slowly, and tend to greatly lessen eventually but do not disappear. Many adults also develop focal palmoplantar hyperkeratosis.

We report six cases of EB-MP in two unrelated Spanish families with several affected members with EBS-MP and review all cases previously reported. In both families, the autosomal-dominant missense p.Pro25Leu mutation in the head domain of KRT5, commonly associated with this subtype, was present in all affected individuals in whom a genetic analysis was performed (4).

\section{CASE REPORTS}

The pedigrees of both families are shown in Fig. 1. 
A

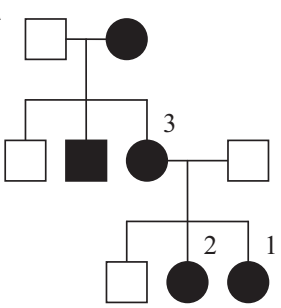

A: Family 1

B: Family 2

Figure 1. Clinical pedigree of three generations of both families.

\section{Family 1}

Patient 1 A 2-month-old girl presented with a history of blisters and a few erosive lesions with crusted surface since birth. The majority of lesions were located on the face (cheeks and perioral region) and frictional areas such as the diaper area, palms, and soles. Lesions healed without scar formation or milia, and the surrounding skin was normal. There was no nail or mucous involvement, and skin color was normal (Fig. 2).

Patient 2 A 2-year-old otherwise healthy girl, the older sister of patient 1 , had a recent history of progressive hyperpigmented lesions. They were asymptomatic brown to tan, confluent, reticulated macules, favoring the axillae and groin, but also appearing on the flexural areas and extremities. She had a past history of nonscarring blisters since birth on both palms and soles, and on physical examination tense bullae on both soles were observed (Fig. 3A,B).
Patient 3 A 30-year-old woman, the mother of the siblings described above, had persistent brownish mottled hyperpigmentation on the groins, armpits, and neck folds and palmoplantar hyperkeratotic papules (Fig. $3 \mathrm{C}, \mathrm{D})$. She did not remember blistering in infancy or early childhood. The older brother of this patient, now 40 years old, was reported to have similar lesions but was unavailable for clinical examination.

\section{Family 2}

Patient 1 A 2-year-old boy presented with a recent history of progressive hyperpigmented, confluent, lesions mostly on his arms and legs. His past history was remarkable for skin fragility and bullae formation since birth.

Patient 2 The mother of patient 1 showed the similar hyperpigmented macules on the extremities, as well as hyperkeratotic papules on both palms and soles. She remembered that her mother had had similar clinical features (Fig. 4A,B).

Patient 3 The cousin of patient 1 had clinically nonscarring blisters on pressure areas and a few erosions (Fig. 4C,D).

\section{HISTOLOGIC AND MOLECULAR ANALYSIS}

A skin biopsy was taken from hyperpigmented lesions on the axilla of patient 3 from family 1 . There were elongated lentiginous rete ridges with some ectatic epidermal follicles and scattered melanin-loaded dermal histiocytes.

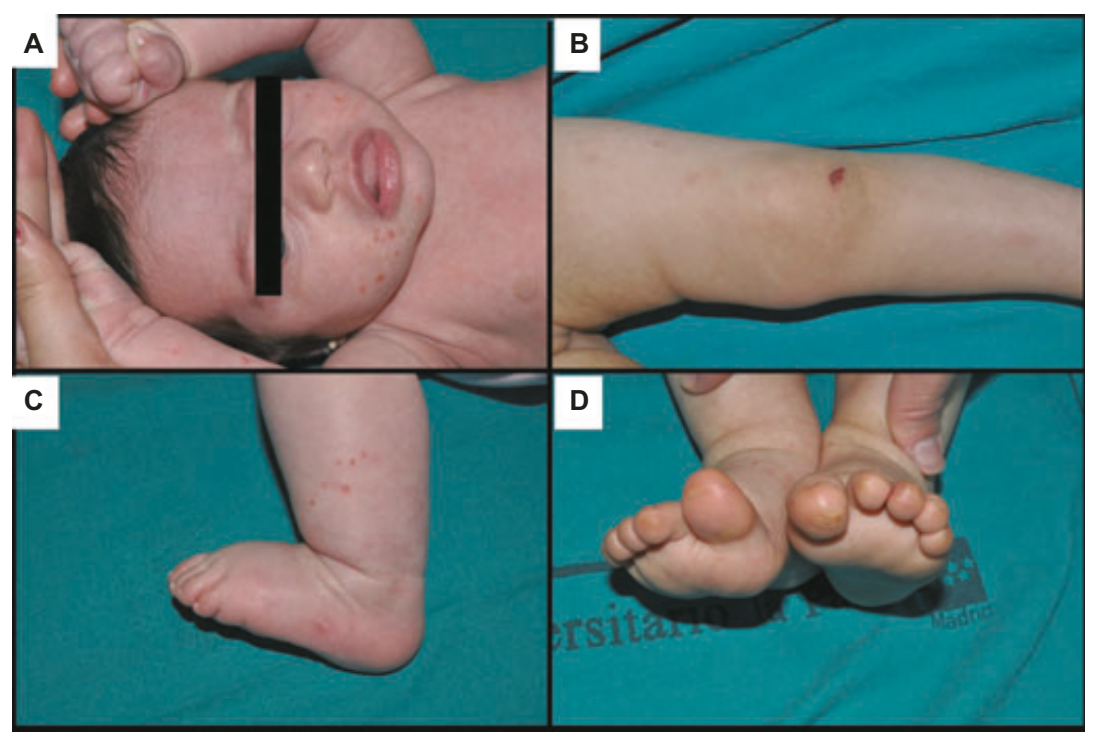

Figure 2. Clinical findings in patient 1 (2-month-old girl), family 1: a few nonscarring perioral blisters (A), erosion on the knee with normal surrounding skin (B), and blisters on the ankle (C) and both feet (D). 


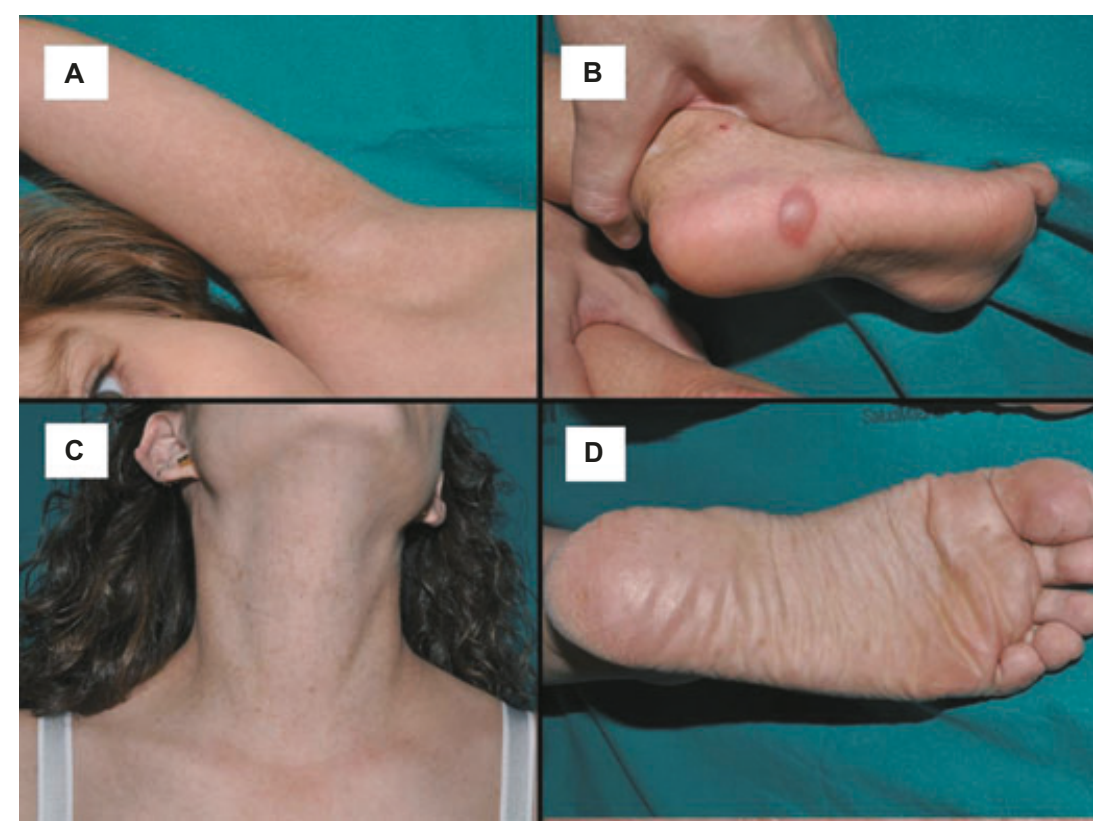

Figure 3. (A,B) Clinical findings in patient 2 (2-year-old girl), family 1. (C,D) Clinical findings in patient 3 (30-year-old woman), family 1 .

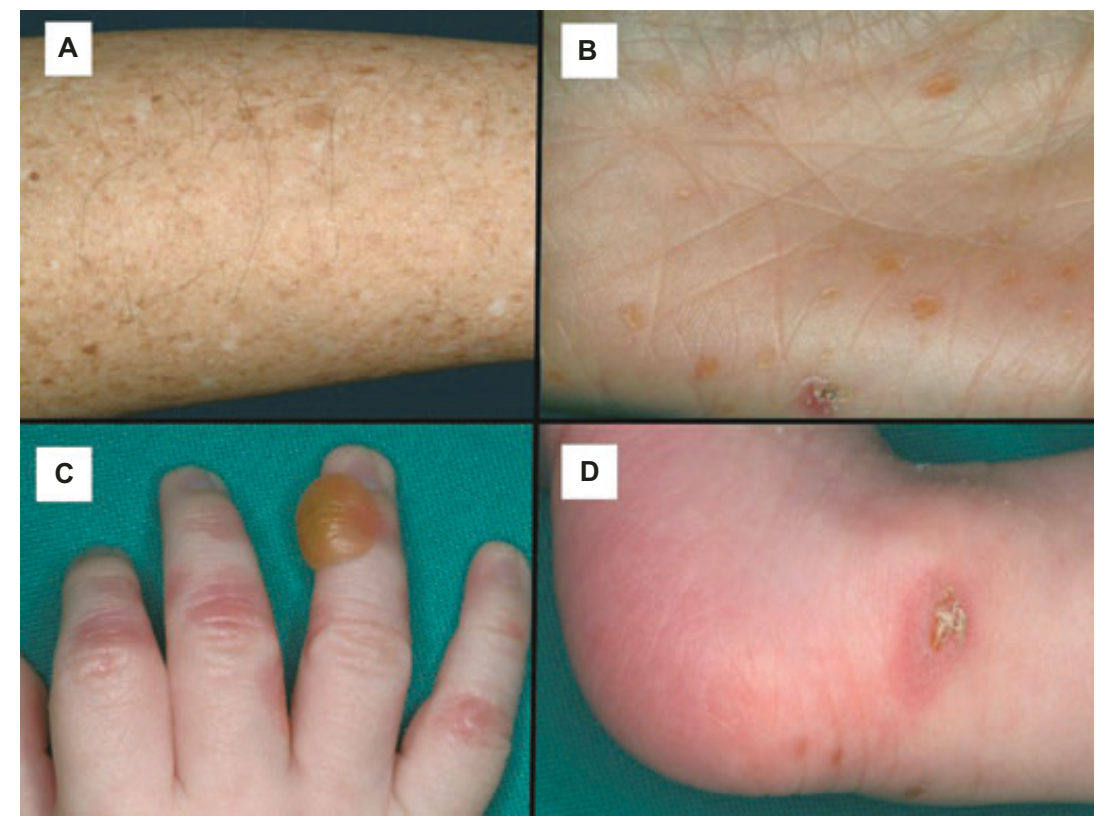

Figure 4. (A,B) Clinical findings in patient 2, family 2 (35-year-old woman). (C,D) Clinical findings in patient 3, family 2 (6-year-old boy).

Genomic DNA (gDNA) from the six patients described herein was screened by direct sequencing for the most common mutation that has been associated with EBSMP (5) KRT5 p.Pro25Leu. In all of the samples, a heterozygous $\mathrm{C}$ to $\mathrm{T}$ transition was detected at nucleotide 74 of the KRT5 gene, resulting in proline to leucine amino acid substitution at the conserved reside 25 of keratin K5.

\section{DISCUSSION}

Clinical manifestations of EBS-MP vary according to patient age, and there is phenotype heterogeneity between members of the same affected family and of different families. Since the first family reported (1), only 15 families and eight sporadic cases of EBS-MP have 
been described (1-22), including the two Spanish families described in the present report (4), (Table 1). In all reported cases for which history is available, the blisters appeared at birth or during infancy and tended to disappear in adulthood, although in some cases they reappeared periodically $(1,6)$. The blisters are usually localized, affecting mainly the distal extremities or acral areas, although generalized blistering has been reported in two families $(1,7)$ and in one sporadic case (3). Blisters heal without scarring, but in the first reported family and in one spontaneous case, physical examination revealed milia $(1,8)$, and in this latter case and in two other reported cases, cutaneous atrophy was also found (9). Some authors $(6,10,11)$ noticed more blisters during summertime, but this situation is not consistently recorded. In our cases, the two siblings of family 1 and the two cousins of family 2 had had blisters since birth that healed without milia or scarring, and in family 1 , the blisters were more generalized in the younger patient than in her older sister, whose blisters faded with age, and appeared only on pressure areas.

Abnormal skin pigmentation appears later in life and is not preceded by blisters. In all reported cases, it is described as hyperpigmented and confluent macules forming a reticular pattern that in some cases may be accompanied by hypopigmented macules $(3,7,12)$. According to previous reports, the most commonly involved areas are the trunk (3,5,6,9,11-15) and the extremities $(1,5,6,10-12,14-17)$, followed by the abdomen $(8,18)$ and the armpits and groin $(2,19)$. In some cases, this hyperpigmentation can progress and involve most of the body surface $(7,20)$.

During adolescence and adulthood, patients may have hyperkeratotic lesions that are most commonly found on the palms and soles. As shown in Table 1, some families lack this clinical feature $(2,3,5,6,10,11,15,18,22)$, whereas the majority of adult members in other families have such lesions $(1,7-9,12-17,19,20)$. In our cases, the siblings were too young to have manifestations of the potential predicted phenotype.

Nail alterations have also been described in some individuals with EBS-MP $(1,3,5,7-9,13,15,16,18,20)$, although it is not a consistent feature. Other uncommon clinical findings were caries $(1,3,9,16)$, found in four cases, and photosensitivity and telangiectasias $(2,3)$, described in only two patients. Finally, in one sporadic case in a 3-year-old boy, attenuated dermatoglyphics on the hands and erosive blepharitis of the left eye were found (10).

Apart from the above-described clinical findings and the family history, antigen mapping and especially molecular analysis are critical to achieving the correct diagnosis (11). Genetically, the mutation most commonly found in EBS-MP is the p.Pro25Leu mutation, which affects the keratin K5 protein (23) (http:// www.interfil.org). This mutation is thought to produce defective keratin filaments that produce an aberrant melanosome uptake, resulting in hyperpigmented areas $(5,14)$. The description of mutations underlying EBSMP distinct to pPro25Leu suggests that other keratin domains might be involved in the process of pigment distribution. Other mutations have been reported. Horiguchi and colleagues (6) described a new mutation in the KRT5 gene (c.1649delG) affecting the tail domain of the keratin K5 (p.Gly550AlafsX77) in two cousins, and Tang and colleagues (18) identified the same mutation in another familiar case. Two mutations associated with the KRT14 gene have also been reported. Harel and colleagues (12) found a heterozygous $\mathrm{T}$ to $\mathrm{C}$ transition at position 356 of the KRT14 gene that results in the substitution of a highly conserved residue (p.M119T), and Arin and colleagues (21) noticed a duplication (c.1117_1158dup42) at the central 2B domain of keratin K14 (p.Ile373Glu386dup). Accordingly, it has been suggested that haploinsufficiency of keratin K5, as reported in patients with another genodermatosis associated with a reticulate pigmentary defect such as Dowling-Degos disease, may alter keratinocyte organization and adhesion, as well as melanosome uptake by keratinocytes and longevity of melanin granules in basal layer cells (24). This hypothesis would explain bullae formation in EBS-MP and the co-occurrence of hyperpigmentation $(5,24)$. Furthermore, it has been postulated that palmoplantar hyperkeratotic lesions in EBS-MP are the consequence of abnormal binding between the keratin filaments of the keratinocytes and the desmosomes (19). The relationship between the rather constant genetic mutations and the variable phenotype is not clearly addressed, and some other modulating genes or environmental factors might play a role in such variable clinical expression.

In children, the main differential diagnosis is other types of EBS, principally the herpetiformis type of Dowling-Meara (EBS-DM; OMIM no. 131760), and Kindler syndrome (KS; OMIM no. 173650) (25). In the EBS-DM with mottled pigmentation the hyperpigmentation seems to be postinflammatory, and the blisters have a typical herpetiform pattern (26). Genetically, mutations at the highly conserved ends (helix initiation and helix termination peptides) of the alpha-helical rod domain of KRT5 and KRT14 genes cause EBS-DM (21). These highly conserved ends are crucial for keratin filament assembly, and their mutations involve a more-generalized and severe phenotype of epidermolysis bullosa. KS is an autosomal-recessive genodermatosis clinically characterized by acral bullae in infancy and early childhood, generalized progressive poikiloderma, 


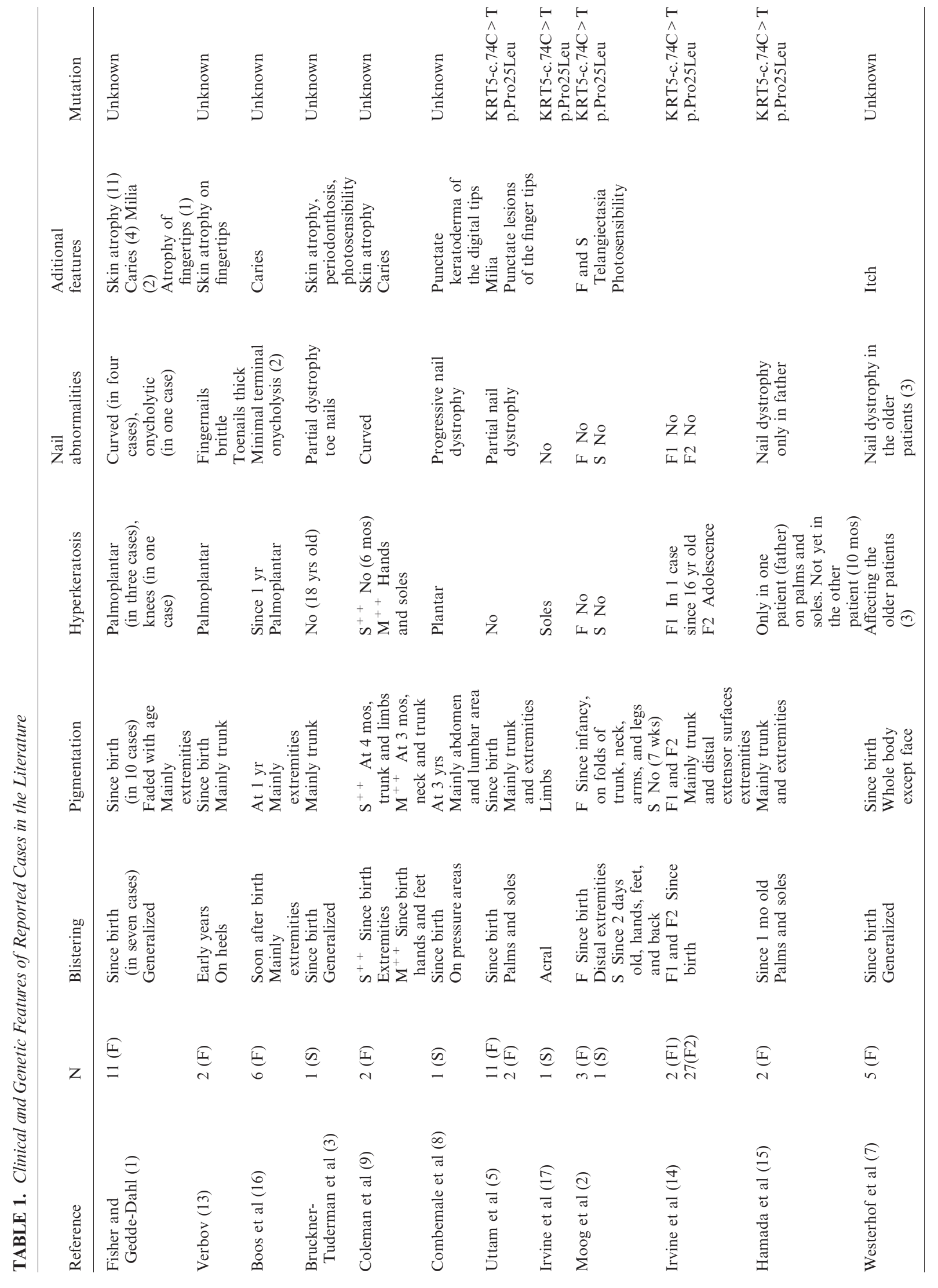




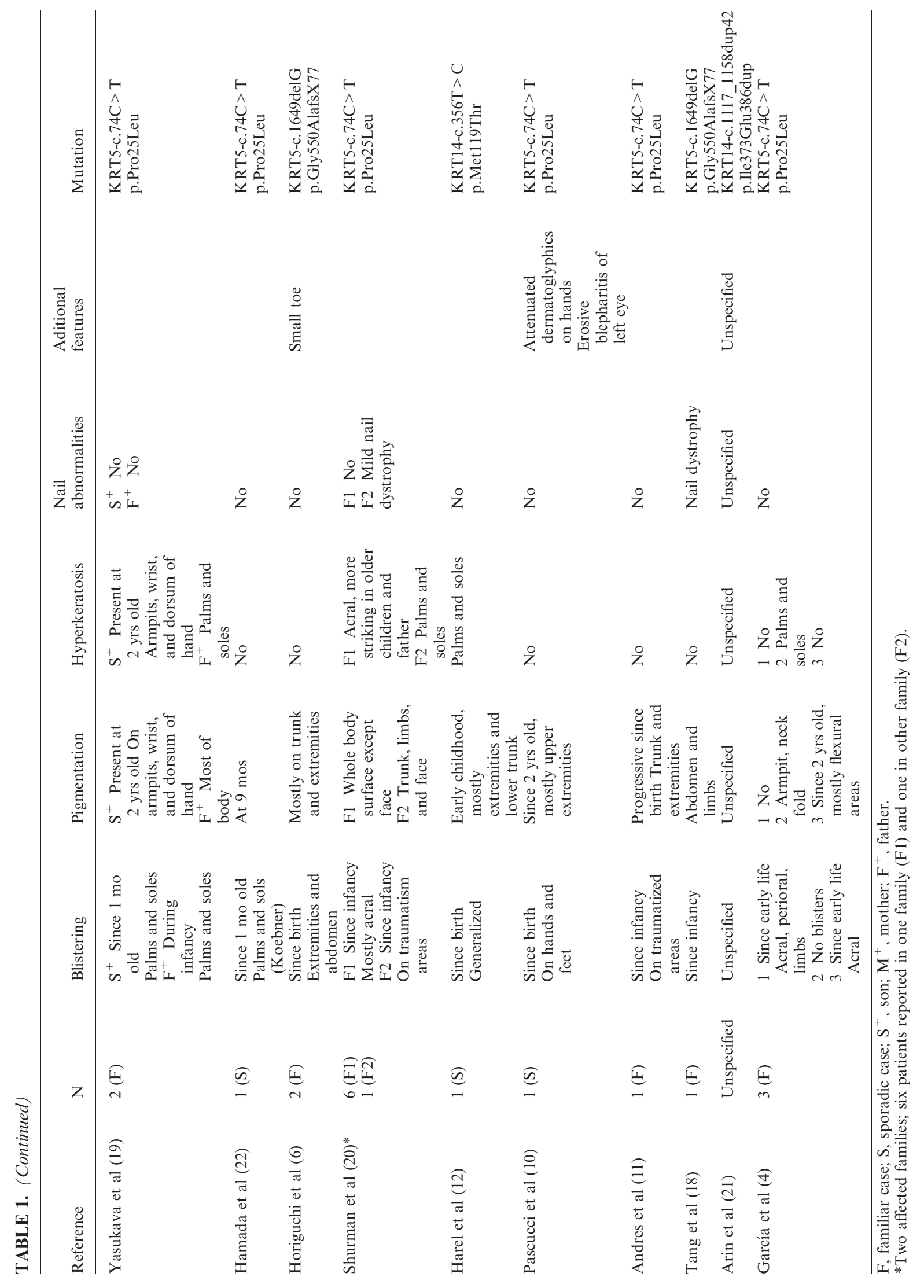


reticulated pigmentation of the neck and face, and diffuse cutaneous atrophy. Genetic testing can be considered to exclude KS, which is caused by a mutation in the FERMT1 (kindlin) gene (27).

In adults, the main differential diagnosis is DowlingDegos disease (DDD; OMIM no. 179850). Mutations in the KRT5 gene are also found in DDD (http://www.interfil.org) (28), but these affect domains other than the rod ends (24). In conclusion, early blistering cannot predict ultimate phenotype. Assessing phenotypic variability, family history and mutation analysis can assist in diagnosis.

\section{REFERENCES}

1. Fischer T, Gedde Dahl T Jr. Epidermolysis bullosa simplex and mottled pigmentation: a new dominant syndrome. I. Clinical and histological features. Clin Genet 1979;15:228 238.

2. Moog U, de Die Smulders CE, Scheffer H et al. Epiderm olysis bullosa simplex with mottled pigmentation: clinical aspects and confirmation of the P24L mutation in the KRT5 gene in further patients. Am J Med Genet 1999; 86:376 379 .

3. Bruckner Tuderman L, Vogel A, Ruegger S et al. Epi dermolysis bullosa simplex with mottled pigmentation. J Am Acad Dermatol 1989;21:425 432.

4. García M, Santiago JL, Terrón A et al. Two novel recessive mutations in KRT14 identified in a cohort of 21 Spanish families with epidermolysis bullosa simplex. Br J Dermatol 2011;165:383 392.

5. Uttam J, Hutton E, Coulombe PA et al. The genetic basis of epidermolysis bullosa simplex with mottled pigmenta tion. Proc Natl Acad Sci U S A 1996;93:9079 9084.

6. Horiguchi Y, Sawamura D, Mori R et al. Clinical heterogeneity of $1649 \mathrm{delG}$ mutation in the tail domain of keratin 5: a Japanese family with epidermolysis bullosa simplex with mottled pigmentation. J Invest Dermatol 2005; 125:83 85.

7. Westerhof W, Dingemans KP. Generalized mottled pig mentation with postnatal skin blistering in three genera tions. J Am Acad Dermatol 2004;50:S65 S69.

8. Combemale P, Kanitakis J. Epidermolysis bullosa simplex with mottled pigmentation. Case report and review of the literature. Dermatology 1994;189:173 178.

9. Coleman R, Harper JI, Lake BD. Epidermolysis bullosa simplex with mottled pigmentation. Br J Dermatol 1993; 128:679 685.

10. Pascucci M, Posteraro P, Pedicelli C et al. Epidermolysis bullosa simplex with mottled pigmentation due to de novo P25L mutation in keratin 5 in an Italian patient. Eur $\mathbf{J}$ Dermatol 2006;16:620 622.

11. Andres C, Chen W, Hofmann $\mathrm{H}$ et al. Epidermolysis bullosa simplex with mottled pigmentation: a case report. Int J Dermatol 2009;48:753 754 .

12. Harel A, Bergman R, Indelman $M$ et al. Epidermolysis bullosa simplex with mottled pigmentation resulting from a recurrent mutation in KRT14. J Invest Dermatol 2006; 126:1654 1657.
13. Verbov J. Hereditary diffuse hyperpigmentation. Clin Exp Dermatol 1980;5:227 234.

14. Irvine AD, Rugg EL, Lane EB et al. Molecular confirma tion of the unique phenotype of epidermolysis bullosa simplex with mottled pigmentation. Br J Dermatol 2001; 144:40 45.

15. Hamada T, Ishii N, Kawano Y et al. The P25L mutation in the KRT5 gene in a Japanese family with epidermolysis bullosa simplex with mottled pigmentation. Br J Dermatol 2004;150:609 611 .

16. Boss JM, Matthews CN, Peachey RD et al. Speckled hyperpigmentation, palmo plantar punctate keratoses and childhood blistering: a clinical triad, with variable associ ations. A report of two families. Br J Dermatol 1981;105: 579585.

17. Irvine AD, McKenna KE, Jenkinson $\mathrm{H}$ et al. A mutation in the V1 domain of keratin 5 causes epidermolysis bullosa simplex with mottled pigmentation. J Invest Dermatol 1997;108:809 810.

18. Tang HY, Du WD, Cui Y et al. One novel and two recurrent mutations in the keratin 5 gene identified in Chinese patients with epidermolysis bullosa simplex. Clin Exp Dermatol 2009;34:e957 e961.

19. Yasukawa K, Sawamura D, Akiyama M et al. Keratotic lesions in epidermolysis bullosa simplex with mottled pigmentation. J Am Acad Dermatol 2005;52:172 173.

20. Shurman D, Losi Sasaki J, Grimwood R et al. Epiderm olysis bullosa simplex with mottled pigmentation: muta tion analysis in the first reported Hispanic pedigree with the largest single generation of affected individuals to date. Eur J Dermatol 2006;16:132 135.

21. Arin MJ, Grimberg G, Schumann $\mathrm{H}$ et al. Identification of novel and known KRT5 and KRT14 mutations in 53 patients with epidermolysis bullosa simplex: correlation between genotype and phenotype. Br J Dermatol 2010; 162:1365 1369.

22. Hamada T, Kawano Y, Szczecinska W et al. Novel keratin 5 and 14 gene mutations in patients with epidermolysis bullosa simplex from Poland. Arch Dermatol Res 2005; 296:577 579.

23. Fine JD, Eady RA, Bauer EA. The classification of inherited epidermolysis bullosa (EB): Report of the Third International Consensus Meeting on Diagnosis and Clas sification of EB. J Am Acad Dermatol 2008;58:931 950.

24. Betz RC, Planko L, Eigelshoven $\mathrm{S}$ et al. Loss of function mutations in the keratin 5 gene lead to Dowling Degos disease. Am J Hum Genet 2006;78:510 519.

25. Prendiville JS, Fine JD, Esterly NB. Kindler syndrome and epidermolysis bullosa simplex. J Am Acad Dermatol 1990;23:327 328 .

26. Tay YK, Weston WL. Epidermolysis bullosa simplex herpetiformis of Dowling Meara with mottled pigmenta tion: the relationship between EBS herpetiformis and EBS with mottled pigmentation. Pediatr Dermatol 1996;13: 306309.

27. Has C, Castiglia D, Del Rio M et al. Kindler syndrome: extension of FERMT1 mutational spectrum and natural history. Hum Mutat 2011;32:1204 1212.

28. Szeverenyi I, Cassidy AJ, Chung CW et al. The Human Intermediate Filament Database: comprehensive informa tion on a gene family involved in many human diseases. Hum Mutat 2008;29:351 360. 